\title{
Significance of Neoadjuvant Downstaging in Carcinoma of Esophagus and Gastroesophageal Junction
}

\author{
S. K. Kamarajah, BMedSci, MBChB ${ }^{1,2}$, M. Navidi, MD FRCSEd ${ }^{1}$, S. Wahed, MD, FRCS ${ }^{1}$, \\ A. Immanuel, MD, FRCSEd ${ }^{1}$, N. Hayes, FRCS $^{1}$, S. M. Griffin, OBE, MD, PRCSEd ${ }^{1}$, and \\ A. W. Phillips, MD, MA, FRCSEd ${ }^{1,3}$ \\ ${ }^{1}$ Northern Oesophagogastric Unit, Royal Victoria Infirmary, Newcastle University Trust Hospitals, Newcastle upon Tyne, \\ UK; ${ }^{2}$ Institute of Cellular Medicine, Newcastle University, Newcastle upon Tyne, UK; ${ }^{3}$ School of Medical Education, \\ Newcastle University, Newcastle upon Tyne, UK
}

\begin{abstract}
Objective. To determine the impact of downstaging on outcomes in esophageal cancer, the prognostic value of clinical and pathological stage, and the difference in survival in patients with similar pathological stages with and without neoadjuvant treatment.

Background. There is little data evaluating adenocarcinoma and squamous cell carcinoma (SCC) and difference in outcomes for similar pathological stage with and without neoadjuvant treatment.

Patients and Methods. Consecutive patients with esophageal cancer from a single center were evaluated. Patients with esophageal adenocarcinoma or SCC treated with transthoracic esophagectomy and two-field lymphadenectomy were included. Comparison of outcomes with those primarily treated with surgery was made. The cTNM and ypTNM 8th edition was used.

Results. This study included 992 patients, of whom 417 received surgery alone and 575 received neoadjuvant therapy and surgery. In the neoadjuvant group, $7(1 \%)$ had cTNM stage 2 and 418 (73\%) had cTNM stage 3. Downstaging rates were similar between adenocarcinoma and
\end{abstract}

Electronic supplementary material The online version of this article (https://doi.org/10.1245/s10434-020-08358-0) contains supplementary material, which is available to authorized users.

(C) The Author(s) 2020

First Received: 11 November 2019; Published Online: 21 March 2020

A. W. Phillips, MD, MA, FRCSEd e-mail: awphillips@doctors.net.uk; alexander.phillips@newcastle.ac.uk
SCC $(54 \%$ vs. $61 \%, p=0.5)$. Downstaging was associated with longer survival than patients with no change (adenocarcinoma, median: 82 vs. 26 months, $p<0.001$; SCC, median: NR vs. 29 months, $p<0.001$ ). On Cox regression analysis, downstaging was associated with significantly longer survival in adenocarcinoma but not in SCC. For SCC and more advanced adenocarcinoma, overall survival was significantly better when comparing like-for-like ypTN to pTN groups.

Conclusions. Pathological stage provides a better estimate of prognosis compared with clinical stage. Downstaged patients may have an improved outcome over those with comparable pathological stage who did not receive neoadjuvant treatment.

Esophageal and gastroesophageal junction (GEJ) cancers are an aggressive disease affecting 450,000 people globally each year. ${ }^{1}$ Currently, neoadjuvant treatment followed by resection is the mainstay treatment of choice in locally advanced cancers, with multimodality approaches shown to improve outcomes. ${ }^{2,3}$ Neoadjuvant therapy improves long-term survival by providing locoregional disease control and reducing the risk of long-term recurrence. ${ }^{4}$ The most important prognostic factor of survival after neoadjuvant therapy followed by surgery is the burden of lymph node involvement. This is reflected in the most recent 8th edition of the American Joint Committee on Cancer (AJCC) and Union internationale contre le cancer (UICC) esophageal TNM staging. ${ }^{5,6}$ The current system grades $\mathrm{N}$-stage from $\mathrm{N} 0$ to $\mathrm{N} 3$ depending on the number of nodes that are involved and has been updated to include clinical, pathological, and post-neoadjuvant staging 
TABLE 1 Demographics for adenocarcinoma and squamous cell carcinoma

\begin{tabular}{|c|c|c|c|c|c|c|c|c|}
\hline & \multicolumn{4}{|c|}{ Adenocarcinoma } & \multicolumn{4}{|c|}{ Squamous cell carcinoma } \\
\hline & Downstaged & No change & Upstaged & $P$ value & Downstaged & No change & Upstaged & $p$ value \\
\hline$n$ & 251 & 188 & 22 & & 69 & 41 & 4 & \\
\hline Age at presentation (years) & $64[58,69]$ & $63[56,69]$ & $60[55,68]$ & 0.548 & $65[58,70]$ & $65[59,68]$ & $72[69,74]$ & 0.094 \\
\hline Gender, male & $220(88)$ & $162(86)$ & $18(82)$ & 0.705 & $31(45)$ & $20(49)$ & $2(50)$ & 0.917 \\
\hline ASA grade & & & & 0.422 & & & & 0.388 \\
\hline Grade 1 & $34(14)$ & $30(16)$ & $2(9)$ & & $14(20)$ & $2(5)$ & $0(0)$ & \\
\hline Grade 2 & $143(57)$ & $107(57)$ & $10(45)$ & & $36(52)$ & $24(59)$ & $3(75)$ & \\
\hline Grade 3 & $64(25)$ & $40(21)$ & $9(41)$ & & $17(25)$ & $13(32)$ & $1(25)$ & \\
\hline Grade 4 & $2(1)$ & $0(0)$ & $0(0)$ & & & & & \\
\hline Unknown & $8(3)$ & $11(6)$ & $1(5)$ & & $2(3)$ & $2(5)$ & $0(0)$ & \\
\hline Overall treatment & & & & 0.513 & & & & 0.046 \\
\hline NAC + surgery & $241(96)$ & $181(96)$ & $20(91)$ & & $46(67)$ & $35(85)$ & $4(100)$ & \\
\hline NACRT + surgery & $10(4)$ & $6(3)$ & $2(9)$ & & $23(33)$ & $6(15)$ & $0(0)$ & \\
\hline NRT + surgery & $0(0)$ & $1(1)$ & $0(0)$ & & - & - & - & \\
\hline Surgical access, thoracic & & & & 0.081 & & & & 0.351 \\
\hline Open & $197(78)$ & $126(67)$ & $19(86)$ & & $58(84)$ & $29(71)$ & $4(100)$ & \\
\hline Thoracoscopic & $4(1)$ & $6(3)$ & $0(0)$ & & $2(3)$ & $1(2)$ & $0(0)$ & \\
\hline Unknown & $50(20)$ & $56(30)$ & $3(14)$ & & $9(13)$ & $11(27)$ & $0(0)$ & \\
\hline Surgical access, abdomen & & & & 0.161 & & & & 0.221 \\
\hline Open & $203(81)$ & $134(71)$ & $19(86)$ & & $61(88)$ & $32(78)$ & $4(100)$ & \\
\hline Laparoscopic & $3(1)$ & $1(1)$ & $0(0)$ & & $2(3)$ & $0(0)$ & $0(0)$ & \\
\hline Unknown & $45(18)$ & $53(28)$ & $3(14)$ & & $6(9)$ & $9(22)$ & $0(0)$ & \\
\hline Overall clinical staging & & & & $<0.001$ & & & & 0.065 \\
\hline Stage 0 & $0(0)$ & $0(0)$ & $1(5)$ & & - & - & - & \\
\hline Stage I & $0(0)$ & $1(1)$ & $0(0)$ & & - & - & - & \\
\hline Stage II & $7(3)$ & $0(0)$ & $0(0)$ & & - & - & - & \\
\hline Stage III & $143(57)$ & $152(81)$ & $21(95)$ & & $58(84)$ & $50(98)$ & $4(100)$ & \\
\hline Stage IV & $101(40)$ & $35(19)$ & $0(0)$ & & $11(16)$ & $1(2)$ & $0(0)$ & \\
\hline Tumor grade & & & & 0.005 & & & & 0.06 \\
\hline Well & $7(3)$ & $5(3)$ & $1(5)$ & & $3(4)$ & $2(5)$ & $1(25)$ & \\
\hline Moderate & $130(52)$ & $78(41)$ & $10(45)$ & & $34(49)$ & $20(49)$ & $2(50)$ & \\
\hline Poor & 97 (39) & $103(55)$ & $11(50)$ & & $14(20)$ & $16(39)$ & $1(25)$ & \\
\hline Unknown & $17(7)$ & $2(1)$ & $0(0)$ & & $18(26)$ & $3(7)$ & $0(0)$ & \\
\hline Lymph nodes examined & $33[25,40]$ & $35[27,44]$ & $33[30,39]$ & 0.08 & $29[24,37]$ & $39[29,42]$ & $32[29,34]$ & 0.004 \\
\hline Margin status, R1 & $1(0)$ & $3(2)$ & $2(9)$ & 0.002 & $0(0)$ & $1(2)$ & $0(0)$ & 0.407 \\
\hline Lymphatic involvement, yes & $98(39)$ & $136(72)$ & $16(73)$ & $<0.001$ & $11(16)$ & $22(54)$ & $4(100)$ & $<0.001$ \\
\hline Venous involvement, yes & $65(26)$ & $98(52)$ & $16(73)$ & $<0.001$ & $6(9)$ & $21(51)$ & $4(100)$ & $<0.001$ \\
\hline Perineural involvement, yes & $83(33)$ & $134(71)$ & $20(91)$ & $<0.001$ & $7(10)$ & $24(59)$ & $4(100)$ & $<0.001$ \\
\hline Tumor regression grade & & & & $<0.001$ & & & & 0.001 \\
\hline 1 & $12(5)$ & $0(0)$ & $0(0)$ & & $12(17)$ & $0(0)$ & $0(0)$ & \\
\hline 2 & $14(6)$ & $2(1)$ & $1(5)$ & & $12(17)$ & $1(2)$ & $0(0)$ & \\
\hline 3 & $53(21)$ & $18(10)$ & $0(0)$ & & $9(13)$ & $4(10)$ & $0(0)$ & \\
\hline 4 & $80(32)$ & $54(29)$ & $12(55)$ & & $12(17)$ & $11(27)$ & $1(25)$ & \\
\hline 5 & $17(7)$ & $10(5)$ & $4(18)$ & & $2(3)$ & $7(17)$ & $2(50)$ & \\
\hline Unknown & $75(30)$ & $104(55)$ & $5(23)$ & & $22(32)$ & $18(44)$ & $1(25)$ & \\
\hline Extracapsular spread, yes & $32(13)$ & $71(38)$ & $19(86)$ & $<0.001$ & $5(7)$ & $15(37)$ & $3(75)$ & $<0.001$ \\
\hline
\end{tabular}

Note that pathological variables such as tumor grade, lymphatic involvement, venous involvement, and perineural involvement do not apply for those who had downstaged disease to pathological complete response (pCR) 
classifications. Whilst its predecessors were based on patients who underwent treatment with surgery alone and did not receive neoadjuvant therapy, ${ }^{6,7}$ the current system uses data from those who have received neoadjuvant treatment, ${ }^{5,6}$ Neoadjuvant therapy has been shown to downstage the initial clinical staging (cTNM) of both tumor invasion $(\mathrm{T})$ and nodal involvement $(\mathrm{N}) .^{8-10}$

Historically, the initial clinical stage of patients with esophageal cancer was thought to determine the outcome. ${ }^{11}$ However, clinical staging is inaccurate, given that only a small proportion of patients will have similar pathological staging, especially in patients with cT3N3 disease. ${ }^{12-14} \mathrm{~A}$ recent study from this institution indicated nodal involvement in approximately $20 \%$ of patients who were originally staged as node negative. More recent evidence has indicated that disease stage following neoadjuvant therapy is a better prognostic marker in patients with adenocarcinoma $^{6,15}$ than initial clinical stage. This has been reflected in the updated TNM staging system. ${ }^{5,6}$ The impact of neoadjuvant therapy on locoregional lymph nodes may therefore have an important bearing on prognosis and help identify patients who would benefit from further adjuvant treatment.

To further improve the current evidence base on the benefit of downstaging, this study aims to determine the impact of lymph node downstaging on the prognosis of patients with esophageal and junctional adenocarcinoma and squamous cell carcinoma (SCC) from a single highvolume center. Furthermore, this study aims to perform a stage-by-stage comparison of pathological staging in patients with (ypTNM) and without (pTNM) neoadjuvant therapy.

\section{PATIENTS AND METHODS}

\section{Patient Population}

Consecutive patients treated for adenocarcinoma or squamous cell carcinoma of the esophagus or gastroesophageal junction between January 2000 and June 2017 from the Northern Oesophagogastric Unit, Newcastle upon Tyne were included. All patients were discussed at a multidisciplinary meeting and subsequently received neoadjuvant chemoradiotherapy followed by transthoracic esophagectomy. Patients were identified from a contemporaneously maintained database.

\section{Pretreatment Staging}

All patients were staged according to standardized protocols which include endoscopy with biopsy, endoscopic ultrasonography, external ultrasonography of the neck (if required), and a thoracoabdominal computed tomography (CT) scan. A positron emission tomography (PET)/CT scan is used in patients being considered for radical (curative) treatment. In patients with histology proven locally advanced resectable malignancy without metastases [cT1N+ or cT3N0-3 or tumors of questionable resectability (cT4)], neoadjuvant chemoradiotherapy followed by surgery is the main treatment option. Patients with a histology other than adenocarcinoma or squamous cell carcinoma or with metastatic disease at the time of the operation were excluded.

\section{Treatment}

Multiple neoadjuvant regimens were employed in the present study, determined by the standard of care and recruiting clinical trials at the time of treatment (Table 1). The majority of patients treated received neoadjuvant chemotherapy. Transthoracic esophagectomy with twofield lymph node dissection was performed within 5-8 weeks after completion of neoadjuvant therapy using a conventional or minimally invasive approach as previously described. ${ }^{16}$

\section{Pathology and Staging}

Histopathological reporting was carried out by specialist gastrointestinal pathologists using a standardized proforma. This was in line with guidelines produced by the Royal College of Pathologists, which included tumor type and differentiation, depth of tumor infiltration, and tumor regression. ${ }^{17}$ Total number of nodes from each location and nodal metastases were recorded along with presence of extracapsular, lymphatic, venous, and perineural invasion.

Lymph nodes were dissected from specimen by the operating surgeon and analyzed separately by the pathologist. ${ }^{18}$ Pathological stage was determined using the AJCC 8th edition TNM staging system. ${ }^{5,6}$

\section{Comparison with Straight-to-Surgery Patients}

In addition to investigating the impact of downstaging, a comparison on outcomes was carried out among patients who underwent surgery but did not receive neoadjuvant chemoradiotherapy. This included a cohort of patients who were pT2N0-neoadjuvant treatment is not routinely offered to patients with pT2N0 disease or earlier. Similar comparisons were made among patients who did not receive neoadjuvant treatment and had a more advanced pathological stage (pT3 N0 vs. ypT3 N0; pT3 N1 vs. ypT3 $\mathrm{N} 1$; pT3/4 N2/3 vs. ypT3/4 N2/3). These patients were discussed at the multidisciplinary meeting (MDM). Neoadjuvant treatment was declined due to concerns 
TABLE 2 Overall survival of esophageal cancers for adenocarcinoma and squamous cell carcinoma

\begin{tabular}{|c|c|c|c|c|c|c|}
\hline & \multicolumn{3}{|c|}{ Adenocarcinoma } & \multicolumn{3}{|c|}{ Squamous cell carcinoma } \\
\hline & $n$ & Median survival, months & $p$ value & $n$ & Median survival, months & $p$ value \\
\hline \multicolumn{7}{|l|}{ All patients } \\
\hline Downstaged $>3$ stages & 52 & $170.1(100.7-\mathrm{NR})$ & \multirow[t]{6}{*}{$<0.001$} & 21 & $90.6(52.8-\mathrm{NR})$ & \multirow[t]{6}{*}{$<0.001$} \\
\hline Downstaged 3 stages & 43 & $147.7(57.2-\mathrm{NR})$ & & 5 & $56.9(56.9-\mathrm{NR})$ & \\
\hline Downstaged 2 stages & 25 & 76.9 (48.9-NR) & & 23 & NR (NR-NR) & \\
\hline Downstaged 1 stage & 131 & $47.7(39.8-85.4)$ & & 20 & NR (33.6-NR) & \\
\hline No change & 188 & $25.9(21.7-30.4)$ & & 41 & $28.8(20.4-46.7)$ & \\
\hline Upstaged & 22 & $18.3(15.4-26.8)$ & & 4 & $19.0(14.2-\mathrm{NR})$ & \\
\hline \multicolumn{7}{|l|}{ T2NO } \\
\hline pT2N0 & 15 & $101.0(61.6-\mathrm{NR})$ & \multirow[t]{2}{*}{0.5} & 4 & 70.7 (49.6-NR) & \multirow[t]{2}{*}{0.001} \\
\hline урT2N0 & 42 & $148.0(74.1-\mathrm{NR})$ & & 21 & NR (NR-NR) & \\
\hline \multicolumn{7}{|l|}{ T3N0 } \\
\hline pT3N0 & 27 & $71.0(40.4-163.0)$ & \multirow[t]{2}{*}{0.2} & 19 & $42.1(13.8-\mathrm{NR})$ & \multirow[t]{2}{*}{0.3} \\
\hline ypT3N0 & 86 & $105.0(59.2-\mathrm{NR})$ & & 23 & $67.5(52.8-\mathrm{NR})$ & \\
\hline \multicolumn{7}{|l|}{$\mathrm{T} 3 / 4 \mathrm{~N} 1$} \\
\hline $\mathrm{pT} 3 / 4 \mathrm{~N} 1$ & 87 & $23.7(16.7-34.7)$ & \multirow[t]{2}{*}{0.5} & 37 & $17.1(13.5-29.2)$ & \multirow[t]{2}{*}{0.048} \\
\hline урT3/4 N1 & 109 & $25.8(20.4-32.6)$ & & 33 & $34.3(23.2-115.7)$ & \\
\hline \multicolumn{7}{|l|}{$\mathrm{T} 3 / 4 \mathrm{~N} 2 / 3$} \\
\hline pT3/4 N2/3 & 21 & $15.9(12.5-24.8)$ & \multirow[t]{2}{*}{0.018} & 3 & 11.5 (7.6-NR) & \multirow[t]{2}{*}{0.2} \\
\hline урT3/4 N2/3 & 84 & $25.8(20.6-31.2)$ & & 15 & $20.3(14.23-46.7)$ & \\
\hline
\end{tabular}

regarding fitness and potential for further deconditioning with neoadjuvant treatment. This could potentially exclude these patients from receiving curative-intent surgery. Further constraints such as inadequate renal and cardiac function excluded patients from receiving neoadjuvant oncological therapy based on local guidelines.

\section{Follow-Up and Definition of Recurrence}

Patients were followed up until death or for 10 years. Patients were seen at 3-6-month intervals in the first 2 years, 6 monthly for 2 years, then annually. After 5 years, follow-up was on a yearly basis. Recurrence of disease was based on clinical grounds and confirmed endoscopically or radiologically.

\section{Definition of Downstaging}

Patients were regarded as having been downstaged if the stage derived from analysis of the pathology specimen was earlier than the clinical stage. Stage movement was regarded as having occurred between any group (e.g., stage IVa to IIIb = 1 stage; stage IVa to IIIa = 2 stages).

\section{Statistical Analysis}

Categorical variables were compared using the Chi squared test. Non-normally distributed data were analyzed using the Mann-Whitney $U$ test. Survival was estimated using Kaplan-Meier survival curves and compared using the log-rank test. Multivariable analyses used Cox proportional hazards models. Stratified survival analyses by underlying histology (adenocarcinoma and squamous cell carcinoma) and by response to neoadjuvant therapy classification were performed. Analyses were also performed according to degree of downstaging ( $>3$ stages, 3 stages, 2 stages, and 1 stage). A $p$ value of $<0.05$ was considered to be statistically significant. Data analysis was performed using R Foundation Statistical software (R 3.2.2) with TableOne, ggplot2, Hmisc, Matchit, and survival packages (R Foundation for Statistical Computing, Vienna, Austria) as previously described. ${ }^{17}$

\section{RESULTS}

\section{Patient Characteristics}

Between January 2000 and June 2017, 992 patients underwent resection for adenocarcinoma or squamous cell carcinoma of the esophagus. Of these, 575 patients 
FIG. 1 Overall survival of patients with esophageal cancer with neoadjuvant therapy:

a adenocarcinoma and

b squamous cell carcinoma
Overall Survival

A

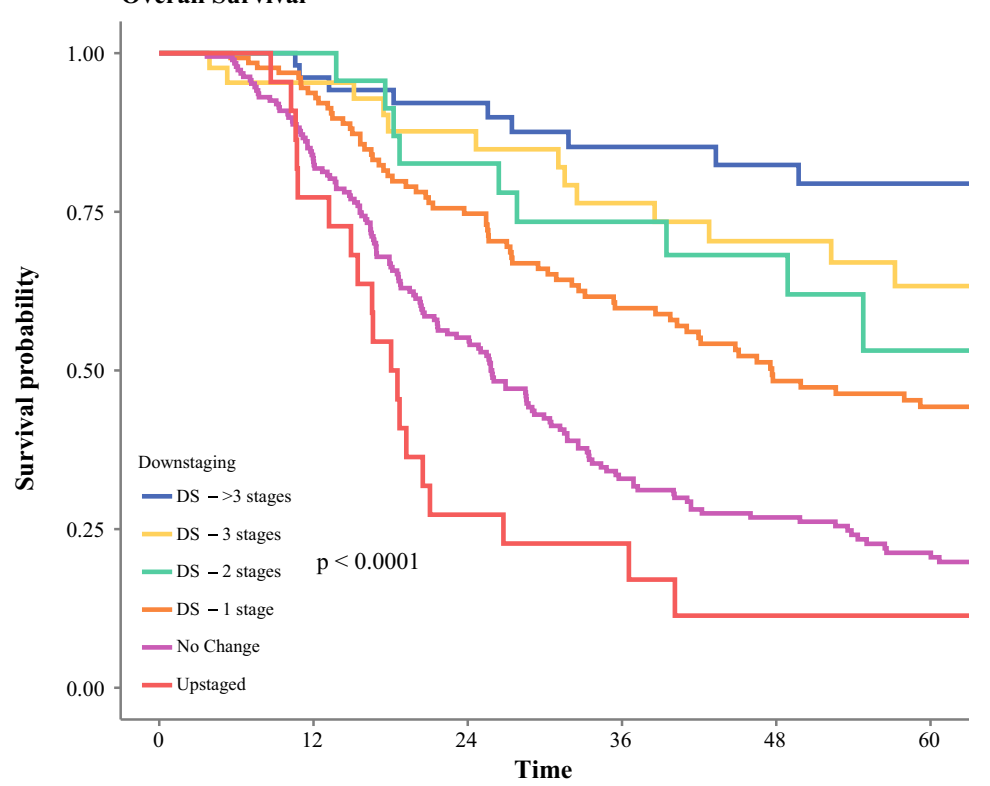

Number at risk

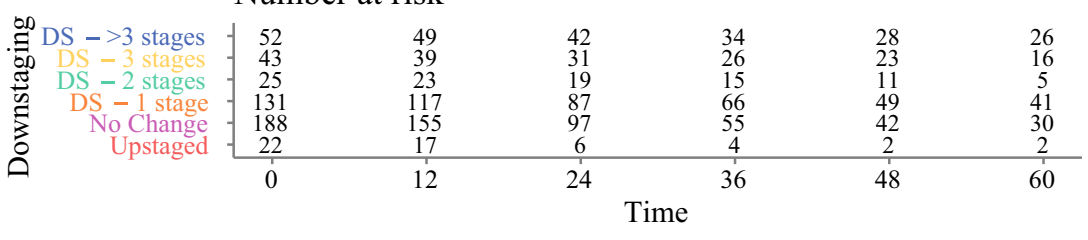

Overall Survival

B

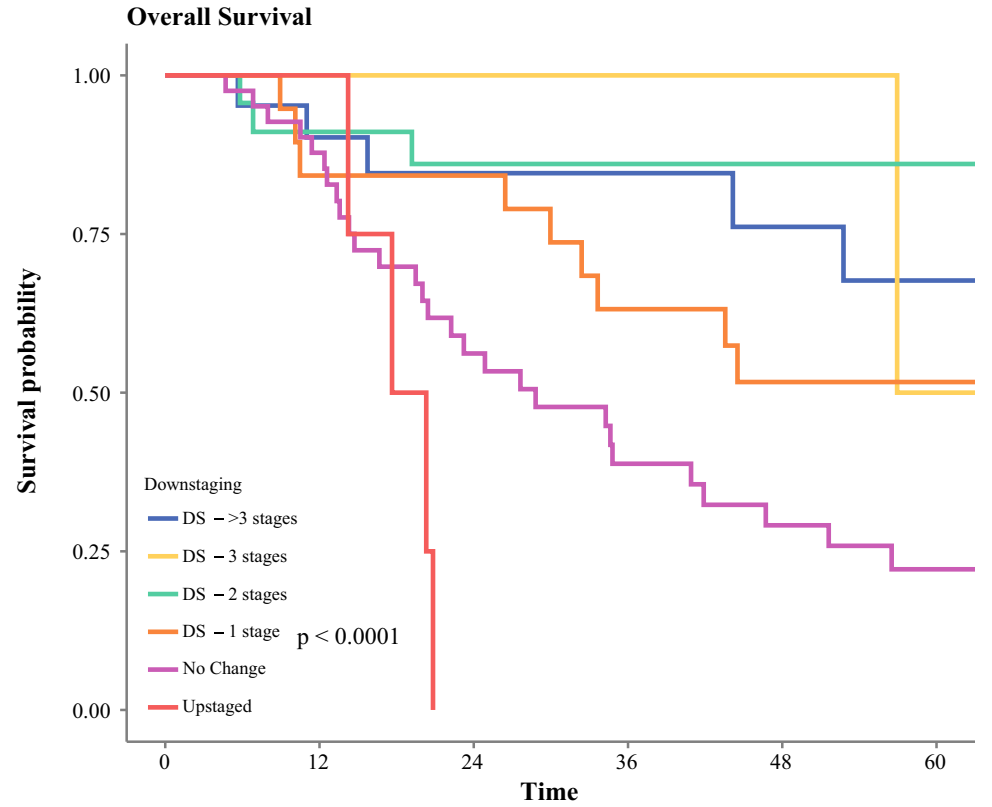

Number at risk
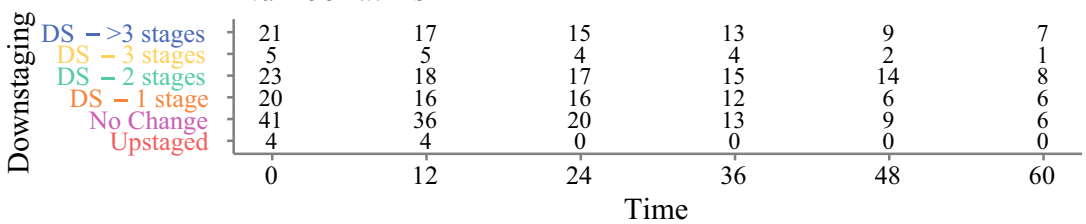

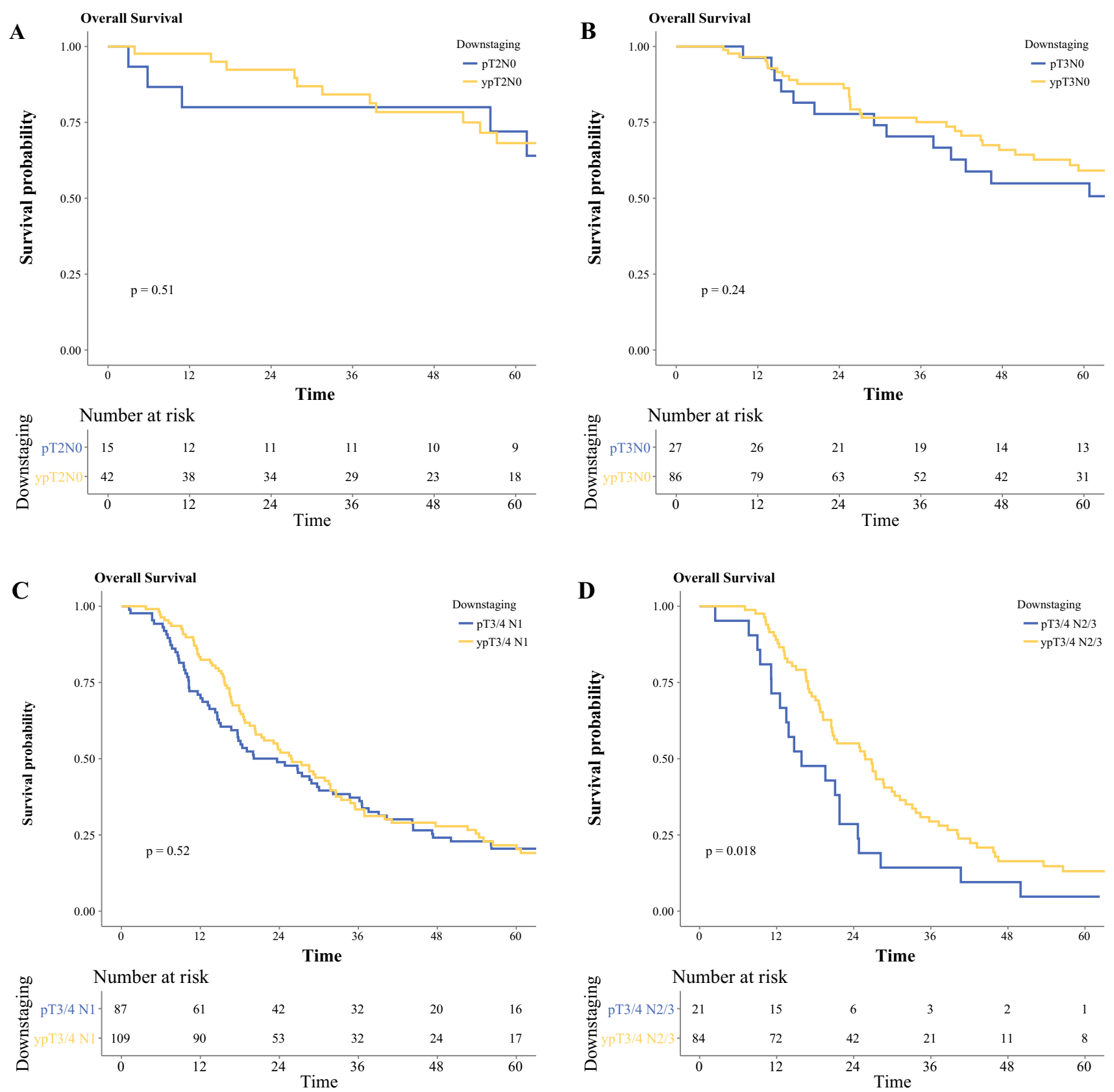

FIG. 2 Stage-by-stage overall survival of patients with esophageal adenocarcinoma with and without neoadjuvant therapy: a T2N0, b T3N0, c T3/4 N1, and d T3/4 N2/3

received neoadjuvant therapy followed by a transthoracic esophagectomy [esophageal adenocarcinoma $(n=461)$; squamous cell carcinoma $(n=114)]$. The remaining 417 patients received unimodality surgery, of whom 8 received adjuvant treatment. Rates of downstaging were higher in patients with SCC than adenocarcinoma, albeit not significantly so $(61 \%$ vs. $54 \%, p=0.5)$ (Table 1$)$. Patients with SCC were more likely to receive neoadjuvant chemoradiotherapy (CRT) compared with adenocarcinoma $(25 \%$ vs. $4 \%, p<0.001)$ and less likely to have lymphatic invasion ( $32 \%$ vs. $54 \%, p<0.001)$, venous invasion $(27 \%$ vs. $39 \%, p=0.028)$, and perineural invasion (31\% vs. $51 \%, p<0.001)$. Patients with SCC had significantly higher rates of TRG 1 compared with adenocarcinoma (11\% vs. $3 \%, p<0.001)$.

\section{Impact of Neoadjuvant Downstaging}

Of patients with adenocarcinoma, $11 \%$ were downstaged by $>3$ stages followed by $9 \%$ and $5 \%$ for 3 stages 

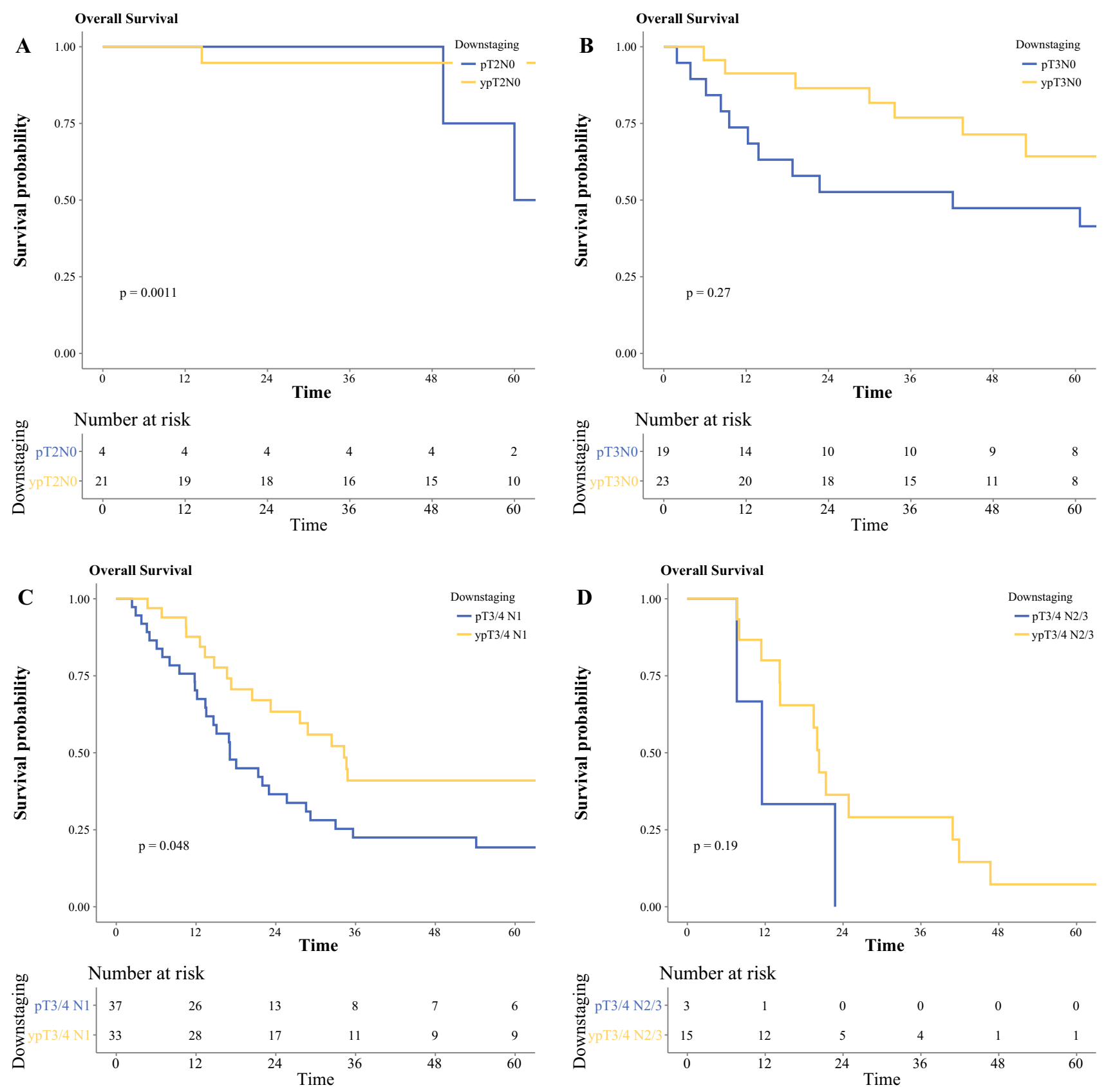

FIG. 3 Stage-by-stage overall survival of patients with esophageal squamous cell carcinoma with and without neoadjuvant therapy: a T2N0, b T3N0, c T3/4 N1, and d T3/4 N2/3

and 2 stages. Patients with downstaged tumors had higher rates of epirubicin, cisplatin, and capecitabine (ECX) chemotherapy regime than those with no change in tumor stage, although not significantly so $(67 \%$ vs. $59 \%$, $p=0.132$ ). Downstaged patients were more likely to have lower rates of lymphatic ( $39 \%$ vs. $72 \%, p<0.001)$, venous ( $26 \%$ vs. $52 \%, p<0.001$ ), and perineural involvement (33\% vs. $71 \%, p<0.001)$ and extracapsular spread $(13 \%$ vs. $38 \%, p<0.001)$ compared with those with no change.
Of patients with SCC, $18 \%$ were downstaged by $>3$ stages followed by $4 \%$ and $20 \%$ for 3 stages and 2 stages. Patients receiving chemoradiotherapy had significantly higher rates of downstaged tumors compared with no change or upstaged (36\% vs. $15 \%$ vs. $20 \%, p=0.041$ ). Downstaged patients were more likely to have lower rates of lymphatic ( $16 \%$ vs. $54 \%, p<0.001)$, venous (9\% vs. $51 \%, p<0.001)$, and perineural involvement (10\% vs. $59 \%, p<0.001)$ and extracapsular spread (7\% vs. $37 \%$, $p<0.001)$ compared with those with no change. 


\section{Overall Survival}

In patients with adenocarcinoma, downstaging was associated with significantly longer survival than no change (median: 82 vs. 26 months, $p<0.001$ ). Patients who were downstaged by $>3$ stages had a significantly longer survival than those downstaged by 3 stages vs. those by 2 stages vs. 1 stage (median: 170 vs. 148 vs. 77 vs. 48 months, $p<0.001$ ) (Table 2; Fig. 1a).

In patients with SCC, downstaging was associated with significantly longer survival than no change (median: NR vs. $29, p<0.001$ ). Patients downstaged by $>3$ stages had a significantly longer survival than those downstaged by 3 stages vs. 2 stages vs. 1 stage (median: 91 vs. 57 vs. NR vs. NR months, $p<0.001$ ) (Table 2; Fig. 1b).

For adenocarcinoma, the multivariable adjusted Cox regression analysis revealed that nonresponder (HR: 1.79, 95\% CI 1.34-2.40, $p<0.001)$, lymphatic involvement (HR: $1.45,95 \%$ CI 1.06-1.98, $p=0.021$ ), perineural involvement (HR 1.57, 95\% CI 1.18-2.09, $p=0.002$ ), and extracapsular spread (HR 1.75, 95\% CI 1.28-2.39, $p<0.001)$ were adverse prognostic factors for overall survival. For squamous cell carcinoma, there were no independent prognostic factors for survival. The Cox regression models are presented in Supplementary Table 1.

\section{Subgroup Analysis by Neoadjuvant Therapy}

To determine the impact of downstaging of neoadjuvant therapy, a stage-by-stage comparison between patients who received neoadjuvant treatment and those neoadjuvant naïve was carried out. During this period, 417 patients did not receive neoadjuvant therapy, of whom $5 \%$ were pT2N0 $(n=19), 11 \%$ were pT3N0 $(n=46), 29 \%$ were pT3/4 N1 $(n=124)$, and $6 \%$ were pT3/4 N2/N3 $(n=24)$.

In patients with adenocarcinoma, there were no significant differences in survival in patients with and without neoadjuvant therapy for T2N0, T3N0, and T3/4 N1 (Fig. 2a-c). However, median survival was significantly longer for ypT3/4 N2/3 compared with pT3/4 N2/3 (median 26 vs. 16 months, $p=0.018$, Fig. 2 d).

In patients with SCC, median survival was significantly longer for ypT2N0 compared with pT2N0 (median: NR vs. 71 months, $p=0.001$ ) and for ypT3/4 N1 compared with pT3/4 N1 (median: 34 vs. 17 months, $p=0.048$ ).

\section{Subgroup Analysis by Downstaging of T3/4 N+}

Supplementary Figs. 1-4 present a more detailed survival analysis of patients initially staged at cT3/4 N + who received chemotherapy stratified for adenocarcinoma and squamous cell carcinoma and who were downstaged to ypT0N0 (Supplementary Fig. 1), ypT1/2 N0
(Supplementary Fig. 2), ypT1/2 N + (Supplementary Fig. 3), or ypT3/4 N0 (Supplementary Fig. 4). In each survival graph, the two control curves represent stagematched patients who were not administered neoadjuvant chemotherapy (pTNM) and patients who were not downstaged by chemotherapy (i.e., nonresponders who were still ypT3/4 N + after surgical resection). In all of these survival analyses, a significant survival benefit was seen in chemotherapy responders versus nonresponders while no difference was observed between responders and stagematched controls (Fig. 3).

\section{DISCUSSION}

Esophageal cancer is an aggressive disease with a high recurrence rate of over $50 \%$ post-neoadjuvant therapy and surgery. ${ }^{19,20}$ These results corroborate what has been found in other recent studies, ${ }^{15,21}$ that disease downstaging with neoadjuvant treatment is associated with better overall survival. This has led to the acceptance of neoadjuvant therapy for locally advanced esophageal cancer as the standard of care. ${ }^{22-24}$ However, it is apparent that not all patients respond to neoadjuvant treatment to the same degree, and the observed impact could be used to tailor adjuvant treatment. In addition, there appeared to be better survival in patients who received neoadjuvant therapy compared with similarly staged neoadjuvant-naïve patients. This was apparent through all the stages for squamous cell carcinoma and evident in more advanced adenocarcinoma.

Current staging of esophageal cancer uses the AJCC 8th edition. This now includes clinical, pathological, and postneoadjuvant stage groupings, allowing their application to clinical practice to be examined. These results highlight the importance of stage post-neoadjuvant treatment for predicting outcomes. To allow accurate staging postsurgery, it is important that adequate lymphadenectomy be carried out. $^{5,6}$ The new staging recognizes a disparity between the lymphadenectomy required to help accurately stage the disease and the lymphadenectomy required for oncological clearance. In the former, this is dependent on tumor size, with short cancers $(<2.5 \mathrm{~cm})$ requiring up to 60 nodes and cancers greater or equal to $2.5 \mathrm{~cm}$ requiring 20 nodes. $^{25}$ To contrast this, the number of nodes required for oncological clearance may be related to the depth of tumor invasion, with T1 tumors needing at least 10 nodes, T2 tumors 20 nodes, and T3 $>30$ nodes. ${ }^{26} \mathrm{~A}$ two-field lymphadenectomy was routinely performed, which ensured that the stage of disease is likely to be accurate with a high median yield in this study (33, range 10-98). ${ }^{27}$

In this study, patients with adenocarcinoma saw a stepwise decline in survival associated with downstaging by more than 3 stages through to those who were upstaged. 
Patients with a 3-stage improvement had a 5-year survival of $60 \%$, while those showing a 1-2-stage improvement had a prognosis of over $36 \%$. In contrast, patients in whom the disease apparently remained static or deteriorated with neoadjuvant treatment had 5-year survival of only $21 \%$ and $13 \%$, respectively. This impact was less pronounced in the squamous cell cohort. This was particularly noticeable after 36 months had passed for SCC patients. Whilst SCC patients who were upstaged or had no change from their clinical stage fared very poorly, initial benefits that seemed apparent disappeared as time from treatment progressed in those with apparent downstaging. It is difficult to determine why this may have happened, but one possibility is a poorer systemic effect for those receiving chemoradiotherapy. This may provide excellent local disease control but have a reduced impact on treating micrometastases.

Approximately $55 \%$ of those with adenocarcinoma and $62 \%$ with squamous cell carcinoma demonstrated significant improvement between clinical stage and posttreatment stage. Overall, over $80 \%$ of patients had adenocarcinoma. Neoadjuvant treatment was generally chemotherapy $(95 \%)$ in these patients. Chemoradiation treatment was used in $28 \%$ of patients with SCC, but only $5 \%$ of those with adenocarcinoma were associated with complete pathological response, approaching $30 \%$ in some studies, with a further $20 \%$ demonstrating significant partial tumor response. $^{28}$ This would reflect the findings from this study, where a significantly higher degree of TRG1 was seen in SCCs, which might reflect the higher proportion receiving chemoradiotherapy. The CROSS study, which evaluated chemoradiation prior to esophagectomy, demonstrated a $23 \%$ complete pathological response, ${ }^{2}$ such levels for chemotherapy prior to surgery have not been reached. ${ }^{29-32}$ Whilst complete response was not evaluated in this study, no significant difference in stage improvement was noted between the use of chemoradiation and chemotherapy. Equally, histological stage had no influence on the degree of downstaging that occurred. Complete pathological response for patients with adenocarcinoma was $4 \%$ compared with $14 \%$ for SCC. Meanwhile, the $\mathrm{SANO}^{33}$ and Esotrate-Prodige ${ }^{34}$ studies seek to determine the impact of watchful waiting in these patients. Previous data have suggested that outcomes of those downstaged are poorer than those with the equivalent "early" disease, ${ }^{35}$ however, these data suggest that this may not be the case. Comparison of outcomes between pTNM and ypTNM demonstrated a significant improvement in survival for patients who received neoadjuvant treatment who were T2 N0 for SCC but not for adenocarcinoma. This impact was also evident for both adenocarcinoma and SCC in the most advanced cancers seen (T3/4 N2/3).
There are a number of limitations to this study that need to be addressed. Comparison of pathological stage with post-neoadjuvant stage may falsely represent the impact of neoadjuvant treatment. Whilst the presumption is that stage migration is due to the effect of neoadjuvant treatment, it is difficult to gauge the full impact given the inherent inaccuracies with staging modalities. ${ }^{36}$ Further, as this study was not a randomized trial, it is potentially susceptible to bias. This is particularly true when comparing outcomes between pathological stage and those who received neoadjuvant chemotherapy. However, overall outcomes represent an unselected consecutive number of patients from a high-volume unit. These patients, though, passed the same MDM and received a standardized two-stage twofield transthoracic esophagectomy, providing a level of quality assurance which some retrospective collaborative studies lack. It appears that both chemotherapy and chemoradiotherapy have a similar impact in downstaging disease and that downstaging confers a definite survival benefit, although this may be different between SCC and adenocarcinoma.

This study reinforces the understanding that postneoadjuvant stage influences prognosis. Whilst some may advocate "complete" restaging prior to progressing to surgery, induction therapy is known to potentially impact on the reliability of staging modalities. ${ }^{32}$ It may also be important to consider the post-neoadjuvant stage when deciding on the merit of adjuvant treatment. There has been some suggestion that adjuvant treatment may confer some benefit in node-positive patients with adenocarcinoma who received neoadjuvant chemoradiotherapy, ${ }^{37}$ and it already forms part of the standard of treatment in patients receiving the MAGIC protocol chemotherapy. ${ }^{11}$

Neoadjuvant oncological therapy with surgery improves survival over surgical intervention alone. Multiple randomized trials have reinforced the superiority of multimodal therapy over surgery alone. ${ }^{11,38,39}$ However, recent studies have demonstrated a deleterious impact of neoadjuvant oncological therapy on fitness. ${ }^{40}$ The impact of this decline on survival has not been established. However, the ability to predetermine the impact of neoadjuvant therapy on disease stage based on specific biological factors of a tumor may further individualize oncological therapy. This may spare a subgroup of patients the adverse physiological impact of neoadjuvant oncological therapy, ${ }^{41}$ in whom, as identified by this paper, neoadjuvant therapy leads to static or worsening stage with no apparent improvement in survival.

In conclusion, this study demonstrates the significant impact of downstaging in patients with both SCC and adenocarcinoma. The optimal neoadjuvant treatment for 
both remains controversial. Understanding and assessment of response to chemotherapy are imperative if patients are to receive individualized treatment.

\section{FUNDING None declared.}

\section{CONFLICT OF INTEREST None declared.}

OPEN ACCESS This article is licensed under a Creative Commons Attribution 4.0 International License, which permits use, sharing, adaptation, distribution and reproduction in any medium or format, as long as you give appropriate credit to the original author(s) and the source, provide a link to the Creative Commons licence, and indicate if changes were made. The images or other third party material in this article are included in the article's Creative Commons licence, unless indicated otherwise in a credit line to the material. If material is not included in the article's Creative Commons licence and your intended use is not permitted by statutory regulation or exceeds the permitted use, you will need to obtain permission directly from the copyright holder. To view a copy of this licence, visit http://creativecommons. org/licenses/by/4.0/.

\section{REFERENCES}

1. Arnold M, Soerjomataram I, Ferlay J, et al. Global incidence of oesophageal cancer by histological subtype in 2012. Gut 2015; 64(3):381-7.

2. van Hagen P, Hulshof MC, van Lanschot JJ, et al. Preoperative chemoradiotherapy for esophageal or junctional cancer. $N$ Engl $J$ Med 2012; 366(22):2074-84.

3. Napier KJ, Scheerer M, Misra S. Esophageal cancer: a review of epidemiology, pathogenesis, staging workup and treatment modalities. World J Gastrointest Oncol 2014; 6(5):112-20.

4. Matsuyama J, Doki Y, Yasuda T, et al. The effect of neoadjuvant chemotherapy on lymph node micrometastases in squamous cell carcinomas of the thoracic esophagus. Surgery 2007; 141(5):570-80.

5. Rice TW, Patil DT, Blackstone EH. 8th edition AJCC/UICC staging of cancers of the esophagus and esophagogastric junction: application to clinical practice. Ann Cardiothorac Surg 2017; 6(2):119-130.

6. Rice TW, Gress DM, Patil DT, et al. Cancer of the esophagus and esophagogastric junction-Major changes in the American Joint Committee on Cancer eighth edition cancer staging manual. $C A$ Cancer J Clin 2017; 67(4):304-317.

7. Rusch VW, Rice TW, Crowley J, et al. The seventh edition of the American Joint Committee on Cancer/International Union Against Cancer Staging Manuals: the new era of data-driven revisions. J Thorac Cardiovasc Surg 2010; 139(4):819-21.

8. Mandard AM, Dalibard F, Mandard JC, et al. Pathologic assessment of tumor regression after preoperative chemoradiotherapy of esophageal carcinoma. Clinicopathologic correlations. Cancer 1994; 73(11):2680-6.

9. Zanoni A. Nodal downstaging in esophageal and esophagogastric junction cancer: more important than ever. J Thorac Dis 2017; 9(7):1839-1842.

10. Davies AR, Myoteri D, Zylstra J, et al. Lymph node regression and survival following neoadjuvant chemotherapy in oesophageal adenocarcinoma. Br J Surg 2018; 105(12):1639-1649.
11. Cunningham D, Allum WH, Stenning SP, et al. Perioperative chemotherapy versus surgery alone for resectable gastroesophageal cancer. N Engl J Med 2006; 355(1):11-20.

12. Kamarajah SK, Newton N, Navidi M, et al. Long-term outcomes of clinical and pathological-staged T3 N3 esophageal cancer. Dis Esophagus 2020. https://doi.org/10.1093/dote/doz109

13. Phillips AW, Kamarajah SK. ASO Author reflections: lymphadenectomy in esophagectomy: Why bother? Ann Surg Oncol 2019; 27(3):701-702.

14. Phillips AW, Hardy K, Navidi $M$, et al. Impact of lymphadenectomy on survival after unimodality transthoracic esophagectomy for adenocarcinoma of esophagus. Ann Surg Oncol 2019; 27(3):692-700.

15. Davies AR, Gossage JA, Zylstra J, et al. Tumor stage after neoadjuvant chemotherapy determines survival after surgery for adenocarcinoma of the esophagus and esophagogastric junction. $J$ Clin Oncol 2014; 32(27):2983-90.

16. Phillips AW, Dent B, Navidi M, et al. Trainee involvement in Ivor Lewis esophagectomy does not negatively impact outcomes. Ann Surg 2018; 267(1):94-98.

17. N M. Dataset for the histopahtological reporting of oesophageal carcinoma. 2007.

18. Lagarde SM, Phillips AW, Navidi M, et al. Clinical outcomes and benefits for staging of surgical lymph node mapping after esophagectomy. Dis Esophagus 2017; 30(12):1-7.

19. Markar SR, Bodnar A, Rosales J, et al. The impact of neoadjuvant chemoradiotherapy on perioperative outcomes, tumor pathology, and survival in clinical stage II and III esophageal cancer. Ann Surg Oncol 2013; 20(12):3935-41.

20. Markar S, Gronnier C, Duhamel A, et al. The impact of severe anastomotic leak on long-term survival and cancer recurrence after surgical resection for esophageal malignancy. Ann Surg 2015; 262(6):972-80.

21. Pennathur A, Luketich JD, Landreneau RJ, et al. Long-term results of a phase II trial of neoadjuvant chemotherapy followed by esophagectomy for locally advanced esophageal neoplasm. Ann Thorac Surg 2008; 85(6):1930-6; (discussion 1936-7).

22. Nieman DR, Peyre CG, Watson TJ, et al. Neoadjuvant treatment response in negative nodes is an important prognosticator after esophagectomy. Ann Thorac Surg 2015; 99(1):277-83.

23. Ott K, Bader FG, Lordick F, et al. Surgical factors influence the outcome after Ivor-Lewis esophagectomy with intrathoracic anastomosis for adenocarcinoma of the esophagogastric junction: a consecutive series of 240 patients at an experienced center. Ann Surg Oncol 2009; 16(4):1017-25.

24. Portale G, Hagen JA, Peters JH, et al. Modern 5-year survival of resectable esophageal adenocarcinoma: single institution experience with 263 patients. J Am Coll Surg 2006; 202(4):588-96; (discussion 596-8).

25. Rice TW, Ishwaran H, Hofstetter WL, et al. Esophageal cancer: associations with (pN+) lymph node metastases. Ann Surg 2017; 265(1):122-129.

26. Rizk NP, Ishwaran H, Rice TW, et al. Optimum lymphadenectomy for esophageal cancer. Ann Surg 2010; 251(1):46-50.

27. Visser E, Markar SR, Ruurda JP, et al. Prognostic value of lymph node yield on overall survival in esophageal cancer patients: a systematic review and meta-analysis. Ann Surg 2019; 269(2):261-268.

28. Merkow RP, Bilimoria KY, McCarter MD, et al. Use of multimodality neoadjuvant therapy for esophageal cancer in the United States: assessment of 987 hospitals. Ann Surg Oncol 2012; 19(2):357-64.

29. Kelsen DP, Ginsberg R, Pajak TF, et al. Chemotherapy followed by surgery compared with surgery alone for localized esophageal cancer. N Engl J Med 1998; 339(27):1979-84. 
30. Ancona E, Ruol A, Santi S, et al. Only pathologic complete response to neoadjuvant chemotherapy improves significantly the long term survival of patients with resectable esophageal squamous cell carcinoma: final report of a randomized, controlled trial of preoperative chemotherapy versus surgery alone. Cancer 2001; 91(11):2165-74.

31. Darnton SJ, Archer VR, Stocken DD, et al. Preoperative mitomycin, ifosfamide, and cisplatin followed by esophagectomy in squamous cell carcinoma of the esophagus: pathologic complete response induced by chemotherapy leads to long-term survival. $J$ Clin Oncol 2003; 21(21):4009-15.

32. Korst RJ, Kansler AL, Port JL, et al. Downstaging of $\mathrm{T}$ or $\mathrm{N}$ predicts long-term survival after preoperative chemotherapy and radical resection for esophageal carcinoma. Ann Thorac Surg 2006; 82(2):480-4; (discussion 484-5).

33. Noordman BJ, Shapiro J, Spaander MC, et al. Accuracy of detecting residual disease after cross neoadjuvant chemoradiotherapy for esophageal cancer (preSANO Trial): rationale and protocol. JMIR Res Protoc 2015; 4(2):e79.

34. Comparison of systematic surgery versus surveillance and rescue surgery in operable oesophageal cancer with a complete clinical response to radiochemotherapy (Esostrate) 2019. https://clinica 1trials.gov/ct2/show/NCT02551458.

35. Donohoe CL, Phillips AW. Cancer of the esophagus and esophagogastric junction: an 8(th) edition staging primer. $J$ Thorac Dis 2017; 9(3):E282-E284.
36. van Vliet EP, Heijenbrok-Kal MH, Hunink MG, et al. Staging investigations for oesophageal cancer: a meta-analysis. $\mathrm{Br} J$ Cancer 2008; 98(3):547-57.

37. Burt BM, Groth SS, Sada YH, et al. Utility of adjuvant chemotherapy after neoadjuvant chemoradiation and esophagectomy for esophageal cancer. Ann Surg 2017; 266(2):297-304.

38. Shapiro J, van Lanschot JJB, Hulshof M, et al. Neoadjuvant chemoradiotherapy plus surgery versus surgery alone for oesophageal or junctional cancer (CROSS): long-term results of a randomised controlled trial. Lancet Oncol 2015; 16(9):1090-1098.

39. Allum WH, Stenning SP, Bancewicz J, et al. Long-term results of a randomized trial of surgery with or without preoperative chemotherapy in esophageal cancer. J Clin Oncol 2009; 27(30):5062-7.

40. Sinclair R, Navidi M, Griffin SM, et al. The impact of neoadjuvant chemotherapy on cardiopulmonary physical fitness in gastrooesophageal adenocarcinoma. Ann $R$ Coll Surg Engl 2016; 98(6):396-400.

41. Navidi M, Phillips AW, Griffin SM, et al. Cardiopulmonary fitness before and after neoadjuvant chemotherapy in patients with oesophagogastric cancer. Br J Surg 2018; 105(7):900-906.

Publisher's Note Springer Nature remains neutral with regard to jurisdictional claims in published maps and institutional affiliations. 